\title{
The premature brain: imaging, anatomy and uncertain outcome
}

\author{
Charles Raybaud
}

Received: 8 July 2013 / Accepted: 10 July 2013

(C) Springer-Verlag Berlin Heidelberg 2013

The saying goes that a child is not a small adult, and certainly an infant is not (only) a small child; a neonate is not an infant, and a fetus is still more different. So what is a premature neonate? At 24 weeks, the brain volume is less than a third of what it would be at term. The interconnection of the cortex with the rest of the gray matter of the central nervous system is only beginning; instead, transient structures like the subplate play the role of a primordial cortex by connecting to both out-going and in-coming axons, before acting as a cortical template by guiding them to their final targets during the last trimester. The various cellular lineages, which constitute the glia - astroglia, oligodendroglia, microglia - are still in a stage of differentiation and migration, while late neurons are still produced by the germinal matrices. At this early stage, the energy requirements of the brain are unknown but likely to be low and quite stable: energy is required only for structural maintenance and growth, but not for function yet, which in the mature brain, will use an estimated $80 \%$ of the energy supply. Accordingly, the vascular system is prominent in the germinal zones only, scarce in the future white matter, and only beginning to develop in the deep layers of the cortex. It is also primitive, made of embryonal channels with underdeveloped walls and essentially no vasomotricity, hardly able to modulate the blood flow and to sustain the perfusion changes secondary to the postnatal mode of cardio-pulmonary circulation.

In term neonates, the gray matter is the most metabolically active part of the brain, therefore making it the most selectively vulnerable to hypoxia-ischemia. For this reason, it is where the lesions are located in case of hypoxic ischemic

This article is part of the special supplement "The Premature Brain"-Guest Editor: Charles Raybaud

C. Raybaud ( $\square)$

Division of Neuroradiology, Hospital for Sick Children, 555

University Avenue, Toronto, ON M5G1X8, Canada

e-mail: charles.raybaud@sickkids.ca injury. In the preterm brain, it is the white matter that is selectively vulnerable to injury because of the developing connectivity, the maturing astroglia and oligodendroglia, and possibly, because of the high density of microglia. This may result in gross lesions like the cystic periventricular leukomalacia, or less immediately apparent alterations like the disrupted maturation process of the oligodendroglia. It appears that the subplate neurons also might be selectively vulnerable, which may compromise the final steps of the connectivity. In addition, the immature vascular system of the preterm infant is exposed to the physiological changes and pathological instability of the cerebral perfusion, which may result in germinal matrix hemorrhages and periventricular hemorrhagic ischemia, and therefore, compromise the late cellular production and destroy the deep white matter. Another pathology that is not well understood but appears to relate to the deep veins as well is reflected on imaging by more or less extensive - and more or less destructive - "punctate lesions." Both periventricular hemorrhages and punctate lesions spare the subplate zone, which belongs to the territory of the cortical veins: this may allow a secondary reorganization of the connectivity with possible partial recovery of function.

The complexity of the development and of the pathogenetic cascades, the potential of plasticity of the developing brain, and the consequent difficulty of prognostication of clinical outcome were the subject of a Research Project at the Hospital for Sick Children, Toronto ON, led by Margot J. Taylor. It was also the topic of the Derek Harwood-Nash Symposium that was held on October 19, 2012, at the Hospital for Sick Children. This special supplement of Neuroradiology collects the contributions of the participants in the symposium, largely based on the results (still incomplete) of this research: Whyte and Blaser and de Vries et al. report on the relative efficacy and limitations of ultrasound and MR in this population of extremely fragile patients. Raybaud et al. correlate the organization of the lesions with the anatomy of the premature forebrain, while Tam focuses on the alterations 
of the cerebellar development. Duerden et al. describe the impact of early resuscitation efforts on the metabolic development. Taylor analyzes the potential of a wide spectrum of advanced morphological and functional MR techniques to assess the premature brain. Pandit et al. specifically discuss the potential of diffusion MR imaging. Sled and NossinManor elaborate on the use of various quantitative methods (morphometry, sulcation, analysis of microstructure, early myelination). Finally, Lee et al. provide and discuss data on the very early development of functional connectivity.

I would like to finish this introduction by thanking all the contributors; the Editorial Board of Neuroradiology led by Guido Wilms, for giving us such an opportunity; Lisa Babinec, the Managing Editor, for her commitment and for solving every problem; and the Editorial Staff at Springer Verlag including Ute Heilmann, for being so helpful and understanding. 